\title{
High-accuracy diagnostic tool for electron cloud observation in the LHC based on synchronous phase measurements
}

\author{
J. F. Esteban Müller, ${ }^{1,2 *}$ P. Baudrenghien, ${ }^{1}$ T. Mastoridis, ${ }^{3}$ E. Shaposhnikova, ${ }^{1}$ and D. Valuch ${ }^{1}$ \\ ${ }^{1}$ CERN, CH-1211 Geneva, Switzerland \\ ${ }^{2}$ EPFL, CH-1015 Lausanne, Switzerland \\ ${ }^{3}$ California Polytechnic State University, San Luis Obispo, 93442 California, USA
}

(Received 17 March 2015; published 12 November 2015)

\begin{abstract}
Electron cloud effects, which include heat load in the cryogenic system, pressure rise, and beam instabilities, are among the main intensity limitations for the LHC operation with $25 \mathrm{~ns}$ spaced bunches. A new observation tool was proposed and developed to monitor the e-cloud activity and it has already been used successfully during the LHC run 1 (2010-2012) and it is being intensively used in operation during the start of the LHC run 2 (2015-2018). It is based on the fact that the power loss of each bunch due to e-cloud can be estimated using bunch-by-bunch measurement of the synchronous phase. The measurements were done using the existing beam phase module of the low-level rf control system. In order to achieve the very high accuracy required, corrections for reflection in the cables and for systematic errors need to be applied followed by a post-processing of the measurements. Results clearly show the e-cloud buildup along the bunch trains and its time evolution during each LHC fill as well as from fill to fill. Measurements during the 2012 LHC scrubbing run reveal a progressive reduction in the e-cloud activity and therefore a decrease in the secondary electron yield. The total beam power loss can be computed as a sum of the contributions from all bunches and compared with the heat load deposited in the cryogenic system.
\end{abstract}

DOI: 10.1103/PhysRevSTAB.18.112801

PACS numbers: 29.20.dk, 29.27.Bd, 29.27.Fh

\section{INTRODUCTION}

At the beginning of the CERN Large Hadron Collider (LHC) run 1 (2009-2013), electron cloud (e-cloud) effects were limiting the LHC operation, leading to an excessive heat load in the cryogenic system, a degradation of the vacuum, transverse instabilities, emittance growth, and particle losses [1,2]. As the e-cloud buildup depends strongly on the bunch spacing, bunch trains with different bunch spacing were injected into both LHC rings (Beam 1 and Beam 2) during the commissioning (see Table I for beam parameters). First, beams with a bunch spacing of $150 \mathrm{~ns}$ were injected, accelerated to $3.5 \mathrm{TeV}$, and brought to collision without any severe e-cloud effect. Then, beams with a bunch spacing of 75 and $50 \mathrm{~ns}$ were injected into the LHC, but the e-cloud effects were stronger and were limiting the number of injections, especially for beams with 50 ns bunch spacing. Scrubbing with beams was proven to be an effective method for reducing the secondary electron yield (SEY) below the e-cloud buildup threshold for $50 \mathrm{~ns}$ beams, although a long time was

\section{jesteban@cern.ch}

Published by the American Physical Society under the terms of the Creative Commons Attribution 3.0 License. Further distribution of this work must maintain attribution to the author(s) and the published article's title, journal citation, and DOI. required ( 2.5 days with $75 \mathrm{~ns}$ beams and 15 days with $50 \mathrm{~ns}$ beams) [3].

Beams with $25 \mathrm{~ns}$ bunch spacing were injected later in 2011, but the strong e-cloud effects were limiting the beam intensity circulating in the ring and quickly degrading the beam quality. Only 60 bunches per beam could be accelerated to $3.5 \mathrm{TeV}$ and collided. At the very end of run 1, after scrubbing with 25 ns beams ( 5 days in 2011 and 6 days in 2012), it was possible to accelerate 804 bunches per beam to $4 \mathrm{TeV}$, but the beams were not collided. The maximum number of bunches collided at $4 \mathrm{TeV}$ was 396 , significantly less than the design number of bunches (2808).

The LHC operation was resumed in April 2015 after the Long Shutdown 1, with an increased top energy of $6.5 \mathrm{TeV}$. The machine commissioning with bunch trains started by injecting bunches spaced by 50 ns. Some e-cloud activity was observed, but e-cloud quickly vanished after a few fills of scrubbing. Then the bunch spacing was reduced to $25 \mathrm{~ns}$ and e-cloud effects reappeared. Two scrubbing runs were scheduled, the first one 9 days long and the second one 14 days long. Although those runs helped in reducing the SEY of the beam pipes, it could not be reduced below the e-cloud build-up threshold.

Following the second scrubbing run of 2015, the LHC started the physics program with $25 \mathrm{~ns}$ beams. The number of bunches injected into the LHC is being increased since that time, but the excessive heat load in the cryogenic 
TABLE I. Summary of parameters at $450 \mathrm{GeV}$ for different beams used during the LHC run 1.

\begin{tabular}{|c|c|c|c|c|c|}
\hline & $150 \mathrm{~ns}$ beam & $75 \mathrm{~ns}$ beam & $50 \mathrm{~ns}$ beam & $25 \mathrm{~ns}$ beam & Nominal \\
\hline Bunch spacing [ns] & 150 & 75 & 50 & 25 & 25 \\
\hline Number of bunches per train & $8 / 12$ & $8 / 24$ & $12 / 24 / 36$ & $24 / 48 / 72$ & 72 \\
\hline Maximum number of bunches & 368 & 936 & 1374 & 2748 & 2808 \\
\hline Bunch intensity $\left[10^{11}\right]$ & $1.1-1.2$ & $1.1-1.2$ & $1.1-1.7$ & $1.1-1.2$ & 1.15 \\
\hline Bunch length $(4 \sigma)[\mathrm{ns}]$ & $1.2-1.6$ & $1.2-1.6$ & $1.2-1.6$ & $1.2-1.6$ & 1.5 \\
\hline Normalized transverse emittance $[\mathrm{mm} \mathrm{mrad}]$ & $2.0-3.5$ & $2.0-3.5$ & $1.5-3.5$ & $1.5-3.5$ & 3.5 \\
\hline
\end{tabular}

system produced by the e-cloud is slowing down the intensity ramp up. At the time of the writing of this paper, the LHC could not be filled completely with the maximum number of bunches yet.

The e-cloud is currently considered to be the main limitation for LHC operation with $25 \mathrm{~ns}$ beams and the deterioration of the beam quality can also cause a reduction of the luminosity delivered to the experiments. Future LHC operation relies on efficient scrubbing of the beam pipe surface to further reduce its SEY and observation tools are required for optimization of the scrubbing strategy and time [4].

In this paper we present a new method for e-cloud observation that uses synchronous phase measurements. The main advantage of this method with respect to the available observations is that it shows the bunch-by-bunch structure of the e-cloud buildup in real time and with sufficient accuracy. A diagnostics tool based on this method is available in the LHC control room and provides useful information for optimization of the scrubbing process. In addition, the heat load can be found and used as a feedforward for the cryogenics control system which has a high inertia. By comparing the synchronous phase measurements with macroparticle simulations the SEY can also be estimated $[5,6]$.

\section{SYNCHRONOUS PHASE SHIFT}

The e-cloud causes an energy loss of the proton beam since the electrons are accelerated by the electric field generated by the beam. The bunch energy loss has been calculated analytically and using simulations [6,7] and found to be dependent on the e-cloud density. Then, the e-cloud buildup can be observed as an increasing bunchby-bunch energy loss along the bunch trains. The bunch energy loss per turn due to e-cloud $U$ is compensated by the rf system and is therefore connected with a phase shift $\Delta \varphi_{e}$ by the following relation:

$$
U=N e V\left[\sin \left(\varphi_{s}+\Delta \varphi_{o}+\Delta \varphi_{e}\right)-\sin \left(\varphi_{s}+\Delta \varphi_{o}\right)\right],
$$

where $N$ is the bunch intensity, $V$ is the amplitude of the $\mathrm{rf}$ voltage, and $\varphi_{s}$ is the synchronous phase in the absence of intensity effects. The phase shift due to other energy loss mechanisms is $\Delta \varphi_{o}$ and, in the LHC, it is defined mainly by the energy loss due to synchrotron radiation and resistive impedance of the ring. The former is the same for all bunches, as it only depends on the proton energy $\left(U \propto E^{4}\right)$, and its contribution to $\Delta \varphi_{o}$ is very small even at $7 \mathrm{TeV}$ $\left(\sim 0.02^{\circ}\right.$ with $\left.V=10 \mathrm{MV}\right)$. The energy loss due to resistive impedance depends on bunch length and distribution, but the associated phase shift is in general also small (less than $0.1^{\circ}$ for nominal beam parameters) [8]. For small $\Delta \varphi_{e}$, Eq. (1) becomes

$$
U \approx N e V \Delta \varphi_{e} \cos \left(\varphi_{s}+\Delta \varphi_{o}\right)
$$

The LHC operates above transition, which implies that $\varphi_{s}=\pi$ in the absence of acceleration and $\pi / 2<\varphi_{s}<\pi$ during acceleration. As the maximum phase deviation from $\pi$ during the acceleration in the LHC is around $4^{\circ}$ and $\Delta \varphi_{o}$ is smaller than $0.1^{\circ} \cos \left(\varphi_{s}+\Delta \varphi_{o}\right) \approx-1$ and Eq. (1) can be replaced by:

$$
U \approx-N e V \Delta \varphi_{e}
$$

Therefore, a negative phase shift $\Delta \varphi_{e}$ indicates an energy loss.

The average bunch power loss $P_{L}$ can be calculated from the bunch energy loss per turn as:

$$
P_{L}=f_{\text {rev }} U
$$

where $f_{\text {rev }}$ is the revolution frequency.

The phase shift due to e-cloud can be extracted from the synchronous phase measured by the beam phase module (PM) from the LHC low-level rf system [9]. It is calculated as the difference between the bunch phase and the rf voltage phase as shown in the simplified scheme in Fig. 1. The bunch phase is computed from longitudinal bunch profiles measured by a wideband pickup (with $3 \mathrm{GHz}$ bandwidth). This signal is fed into a strip-line comb filter transforming a single pulse (a bunch profile) into a wavelet at $400.8 \mathrm{MHz}$ lasting for $9 \mathrm{rf}$ periods. The filter output is the beam signal input to the PM. The second rf input is the vector sum of the eight cavity-antenna signals. Appropriate delays are added to the cavity signals before addition to compensate for the time of flight between them. Two analog in-phase/quadrature $(I / Q)$ demodulators transform the bunch signal and cavity sum into $(I, Q)$ pairs and an 


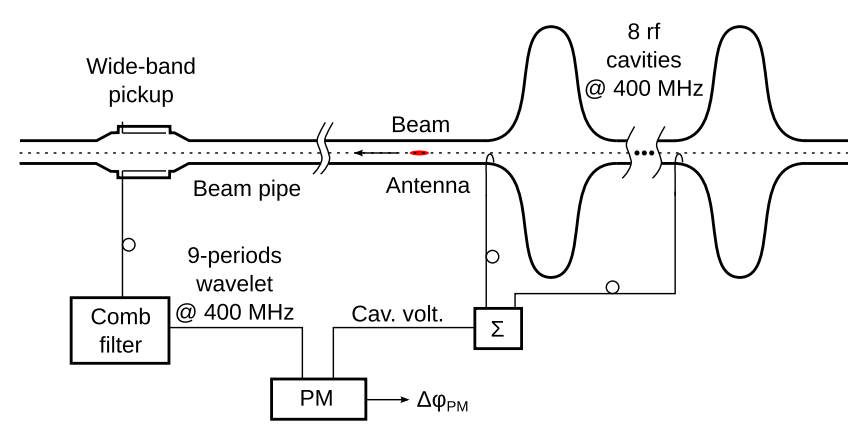

FIG. 1. Simplified scheme of the synchronous phase measurement. A wavelet is generated from the wideband pickup signal and is compared in phase in the beam phase module with the vector sum of the voltages of the eight rf cavities.

FPGA computes the phase and amplitude of both signals (see Appendix A). The effect of beam loading is excluded by measuring the real voltage in the cavities (for details see Appendix B).

The PM is capable of measuring the bunch amplitude and phase, phase shift, as well as cavity voltage amplitude $V$ for buckets spaced by $25 \mathrm{~ns}$ over the whole ring, and in bursts of 73 acquisitions at an adjustable rate (memory limit of the PM), which is usually set to 5 revolution periods $(0.45 \mathrm{~ms})$. A typical burst therefore covers $33 \mathrm{~ms}$, almost 2 synchrotron periods $T_{s}$ at $450 \mathrm{GeV}\left(T_{s}=18 \mathrm{~ms}\right.$ for $V=6 \mathrm{MV}$ ). During phase measurements, a burst is usually acquired every $\sim 10-15 \mathrm{~s}$.

The resulting phase measured by the $\mathrm{PM}\left(\Delta \varphi_{\mathrm{PM}}\right.$ in Fig. 1) is therefore defined as:

$$
\Delta \varphi_{\mathrm{PM}}=\varphi_{s}+\Delta \varphi_{o}+\Delta \varphi_{e}+\varphi_{\mathrm{off}},
$$

where $\varphi_{\text {off }}$ is an unknown phase offset due to the different delays of the pick-up and voltage signals, and the time of flight from the cavities to the pick-up. In order to obtain
$\Delta \varphi_{e}$, it is necessary to define the other terms of Eq. (5). Both phases $\varphi_{s}$ and $\varphi_{\text {off }}$ are the same for all bunches and the difference in the phase shift $\Delta \varphi_{o}$ from bunch to bunch is in general small (less than $0.1^{\circ}$ see above). Therefore, $\Delta \varphi_{e}$ can be computed as the phase relative to the first bunch train, which in normal operation is shorter (12 bunches for $25 \mathrm{~ns}$ beams and 6 bunches for $50 \mathrm{~ns}$ beams) and has the beam abort gap in front of it ( $3 \mu \mathrm{s}$ without beam), thus is practically not affected by e-cloud $\left(\Delta \varphi_{e} \approx 0\right)$.

The rf phase loop averages the synchronous phase of all bunches measured by the PM and uses this average value to control the rf phase. The average phase is stored in the CERN logging database [10] in normal rf operation and, for that reason, it was the first signal used for e-cloud observations, as described in the next section. Later a bunch-by-bunch observation tool was developed and it is described in Sec. IV. Examples of the data obtained using the PM of Fig. 1 are presented in Fig. 2 (average phase) and in Fig. 3 (raw bunch-by-bunch phase).

A new system is being commissioned during the LHC run 2 and will provide more accurate measurements of the synchronous phase. The improvements with respect to the current system will be discussed in the following sections.

Bunch positions measured by the beam quality monitor (BQM) [11] were also considered to extract the synchronous phase shift [12], but this method could not be used because the phase shift due to transient beam loading is included and it can be larger than the phase shift due to e-cloud (for details see Appendix C).

\section{AVERAGE SYNCHRONOUS PHASE}

The first e-cloud observations based on synchronous phase measurements were done using the average phase $\left\langle\Delta \varphi_{\mathrm{PM}}\right\rangle$, as this signal was available in the logging database. The total beam energy loss per turn $U_{T}$ is the sum of the energy loss of each bunch defined by Eq. (3):

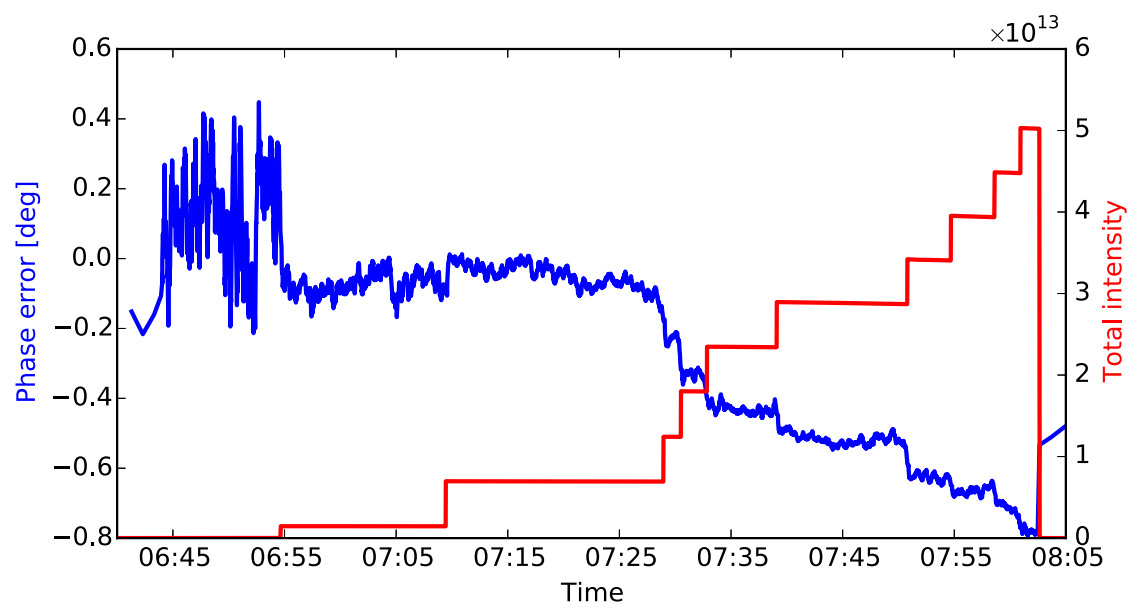

FIG. 2. Example of average phase shift (blue) and total beam intensity (red) of Beam 1 at the LHC flat bottom (450 GeV) during the injection of trains of 48 bunches spaced by $50 \mathrm{~ns}$ from the SPS. Note the phase change after each bunch train injection. $\bar{N} \sim 1.1 \times 10^{11}$. Fill 1502 (20-11-2010). 


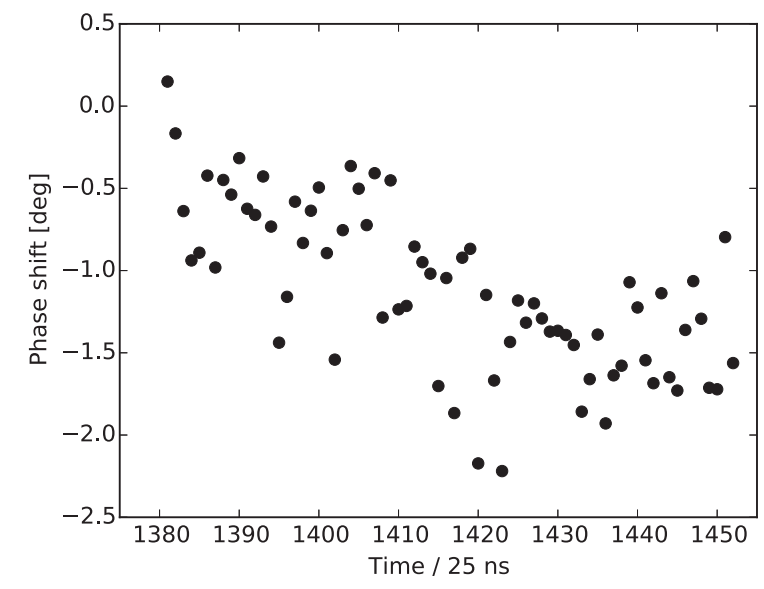

FIG. 3. Example of raw data for the phase shift along a bunch train of 72 bunches with $\bar{N} \sim 1.1 \times 10^{11}$ averaged over 73 acquisitions spaced by 5 turns. Measurements were taken on Beam 1 at injection energy at the beginning of the 2012 scrubbing run with 25 ns spaced bunches (Fill 3389, 6-12-2012).

$$
U_{T}=\sum_{k=1}^{M} U_{k} \approx-\sum_{k=1}^{M} e V N_{k} \Delta \varphi_{e k},
$$

where $M$ is the total number of bunches.

For bunches with a small variation from the average bunch intensity $\bar{N}=\sum_{k=1}^{M} N_{k} / M$, i.e., $N_{k}=\bar{N}+\Delta N_{k}$ and $\Delta N_{k} \ll \bar{N}$, the total beam energy loss per turn can be approximated as:

$$
U_{T} \approx-e V M \bar{N}\left\langle\Delta \varphi_{e}\right\rangle
$$

where the average phase shift due to e-cloud $\left\langle\Delta \varphi_{e}\right\rangle$ can be calculated using Eq. (5) as:

$$
\left\langle\Delta \varphi_{e}\right\rangle=\left\langle\Delta \varphi_{\mathrm{PM}}\right\rangle-\left\langle\Delta \varphi_{o}\right\rangle-\varphi_{s}-\varphi_{\text {off }} .
$$

Figure 2 shows an example of an LHC fill with a strong e-cloud effect which gave an additional shift of the average phase at each injection. This means that the beam energy loss per turn and per particle was increasing with total beam intensity in the ring, suggesting a higher e-cloud density after the injection of a new SPS bunch train (e-cloud build-up).

An analysis of several fills with 50 ns beams during the LHC scrubbing run in April 2011 is presented in Fig. 4. The average phase shift due to e-cloud at each injection is presented as a function of the total beam intensity circulating at that moment. The phase difference between $\left\langle\Delta \varphi_{e}\right\rangle$ and $\left\langle\Delta \varphi_{\mathrm{PM}}\right\rangle$ is taken into account by performing a linear fit of the total beam intensity against the average phase. The slopes of these curves, shown in Fig. 5, are related to the e-cloud density and therefore the decrease in the slopes during the scrubbing run is a sign of a SEY reduction.

Similar results were obtained for fills with $75 \mathrm{~ns}$ beams at the beginning of the LHC run 1 and later with $25 \mathrm{~ns}$ beams.

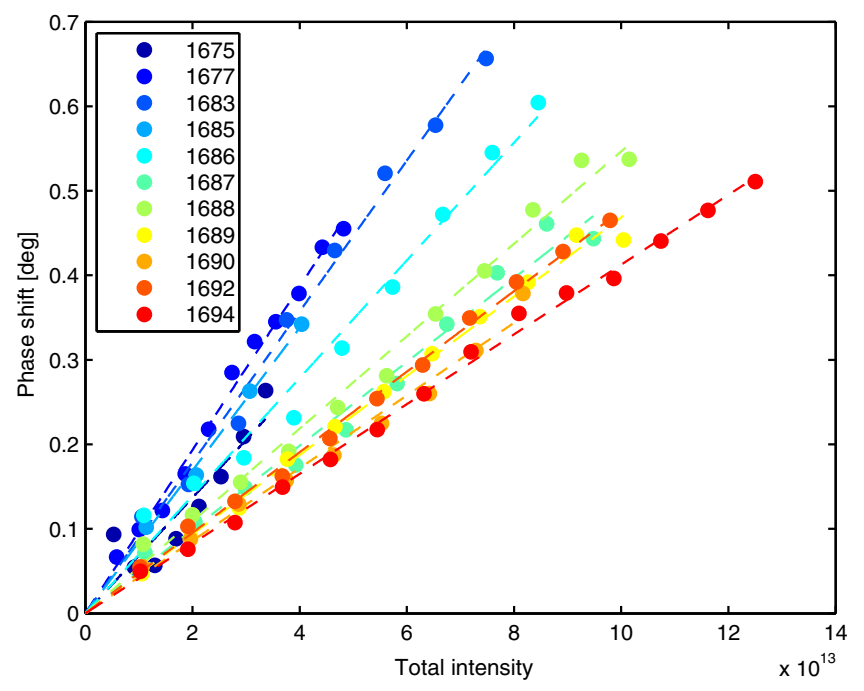

FIG. 4. Average phase shift at bunch train injections in the LHC as a function of the total beam intensity in Beam 1 for the $50 \mathrm{~ns}$ beams during the scrubbing run in April 2011. The decrease in the slope of the curves between the first and the last fills is a sign of surface scrubbing. Each color corresponds to a different LHC fill. $\bar{N} \sim 1.2 \times 10^{11}$.

In Fig. 5 one can also see that after the scrubbing run in April 2011, the slopes were similar for 75 and 50 ns beams, meaning that the SEY was reduced below or very close to their e-cloud build-up threshold. That was not the case for the $25 \mathrm{~ns}$ beams even after the 2012 scrubbing run.

The use of the average phase shift for e-cloud observations has a few limitations that must be taken into account. Usually, e-cloud causes particle losses that are higher at the

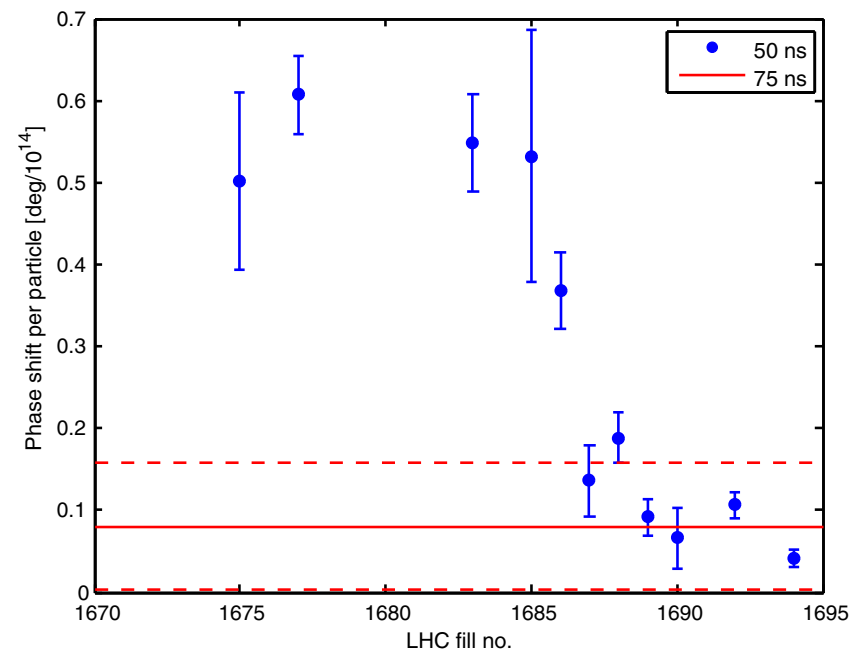

FIG. 5. Ratio of the phase shift to total beam intensity for different LHC fill with $50 \mathrm{~ns}$ beams (blue circles) and mean value (solid red line) together with standard deviation (dashed red lines) of the same ratio for the $75 \mathrm{~ns}$ beams. After scrubbing run in April 2011 the value of the ratio for the $50 \mathrm{~ns}$ beams converged to the 75 ns beams value. 


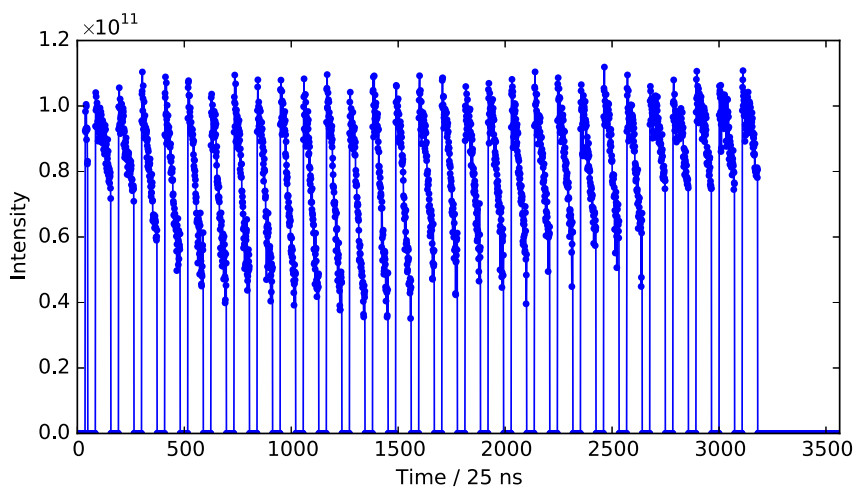

FIG. 6. Typical bunch intensity pattern for the 25 ns beams, with triangular bunch trains due to particle loss from the e-cloud effect. The last five bunch trains were injected later and they were still losing particles. Beam 1. Fill 2251 (25-10-2011).

end of the bunch trains, as shown in Fig. 6. Under these circumstances the uniform bunch intensity approximation used in Eq. (7) is not valid anymore. Another limitation is due to thermal drifts of the phase that cannot be distinguished from those caused by changes in the e-cloud density (e.g., due to particle losses). In fact, the data for Beam 2 had inexplicably larger drifts, so that only the average phase measurements for Beam 1 are valid. In addition, the average phase can be affected by the errors in the bunch-by-bunch measurements discussed in the next section.

\section{BUNCH-BY-BUNCH SYNCHRONOUS PHASE}

In order to improve the accuracy of the e-cloud observation and to avoid the limitations described above, a new tool was developed allowing the acquisition of the bunchby-bunch phase from the same phase module. Despite the very high accuracy of the PM (around $1^{\circ}$ at $400 \mathrm{MHz}$ ), the noise of the raw data is comparable to the phase shift due to e-cloud (see Fig. 3). An accuracy of $\sim 0.1^{\circ}$ is required to resolve e-cloud caused phase shifts. Two corrections were applied to the measurements to minimize systematic errors together with a post-processing, which improves the precision, and they are described below.

\section{A. Measurement corrections}

After a bunch passage, reflections due to impedance mismatch in the signal transmission path affect subsequent bunch phase measurements. For high accuracy results, it is necessary to remove these perturbations. The impulse response of the system from the pickup to the PM (see Fig. 7, blue trace) was measured with a single bunch and then used for correction of the multibunch data.

A new phase module was installed in the underground LHC Faraday Cage during the Long Shutdown 1 and timedomain reflectometry measurements were done to identify possible sources of reflections. The signal routing was

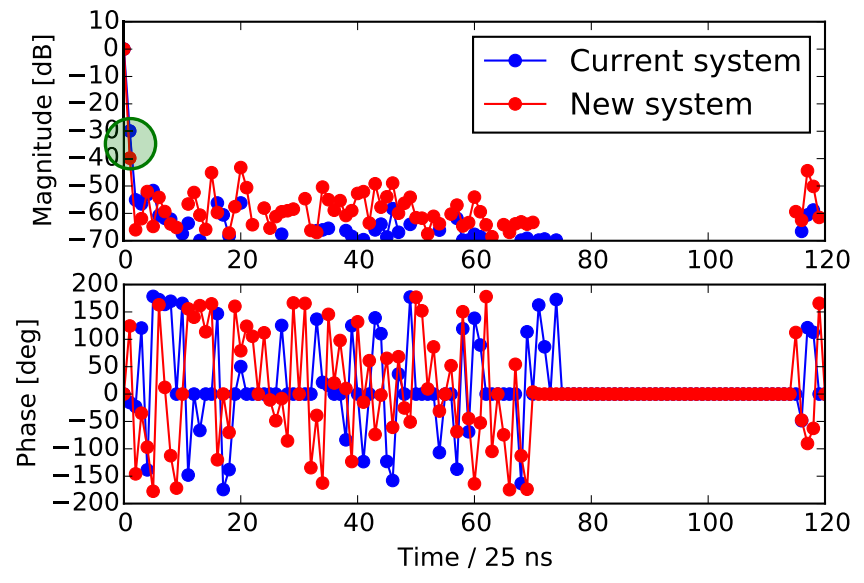

FIG. 7. Impulse response from the pick-up to the PM of the current system (blue) and of the new system (red), measured with a single bunch during $3 \mu \mathrm{s}$. Note that the strongest reflection affects the following bunch (bucket position 1 in these plots, green circle) and in the new system it is reduced by $\sim 10 \mathrm{~dB}$. Reflections below $-70 \mathrm{~dB}$ are neglected.

optimized, improving the impedance match at the pickup output and using shorter cables, with the aim of reducing the reflections and minimizing the signal distortion. The impulse response of the new system is shown in Fig. 7 (red trace) and a clear reduction of the first reflection can be observed.

Another correction applied to the raw data helps to minimize a systematic error which is introduced by the residual offsets of the $I$ and $Q$ components $\left(I_{o}\right.$ and $Q_{o}$, respectively) of the bunch signal (see also Appendix A). The diagram in Fig. 8 shows that the error $\delta \varphi$ in the phase measurement depends on the offsets and on the amplitude $A$ and phase $\varphi_{\mathrm{PM}}$ of the bunch signal as:

$$
\delta \varphi=-\tan ^{-1}\left[\frac{A_{o} \sin \left(\theta_{o}^{\prime}\right)}{A-A_{o} \cos \left(\theta_{o}^{\prime}\right)}\right],
$$

where $\theta_{o}^{\prime}=\varphi_{\mathrm{PM}}-\theta_{o}, \quad \theta_{o}=\tan ^{-1}\left(Q_{o} / I_{o}\right), \quad$ and $A_{o}=$ $\sqrt{I_{o}^{2}+Q_{o}^{2}}$.

The values of $I_{o}$ and $Q_{o}$ are estimated by reconstructing the $I Q$ plane from the bunch amplitude and phase signals and averaging the noise measured in the empty buckets (assuming white noise). The standard deviation of this noise can be used to estimate the remaining error of the bunch phase measurements. For a bunch intensity of $1.1 \times 10^{11}$, the noise amplitude after corrections is typically $\sim 1 / 700$ of the bunch amplitude signal. In the worst case, which can happen when the angle between the noise and the bunch signals is $90^{\circ}$, it would lead to a phase error of $\pm 0.08^{\circ}$. The phase shift after these corrections is shown in Fig. 10 (blue squares). 


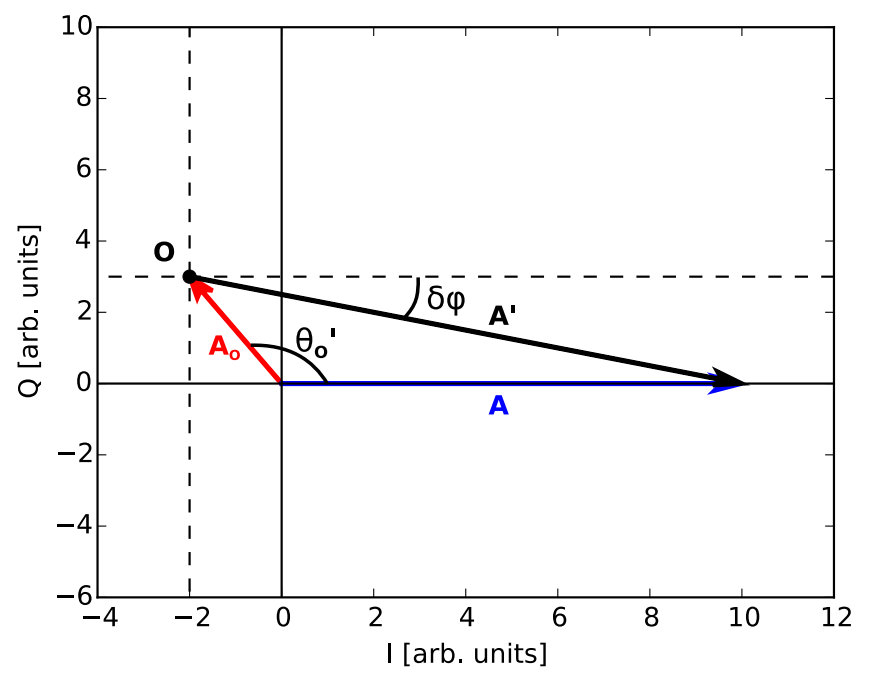

FIG. 8. Vector representation of the error introduced by the residual offsets of the $I$ and $Q$ components of the bunch signal for the case $\varphi_{\mathrm{PM}}=0$. The original vector $\mathbf{A}^{\prime}$ (black) is defined from the real origin $\mathbf{O}$ to the end of the measured vector $\mathbf{A}$ (blue). The phase error $\delta \varphi$ is the angle between $\mathbf{A}^{\prime}$ and $\mathbf{A}$, and it depends on $|\mathbf{A}|,\left|\mathbf{A}_{\mathbf{o}}\right|$ and $\theta_{o}^{\prime}$ as defined in Eq. (9).

\section{B. Data post-processing}

The data post-processing significantly improves the measurement precision and involves two steps that are applied to each bunch separately. First, the variation of the phase shift in the 73 acquisitions is checked for each burst. If it is smaller than $1^{\circ}$ we assume that the bunch is not oscillating and the value of the phase shift can be calculated as the average of the 73 acquisitions. In this case, the maximum error would be in the range $\pm 0.5^{\circ} / \sqrt{73}=$ $\pm 0.06^{\circ}$, which is acceptable when compared to the typical phase shift due to e-cloud (in the order of $1^{\circ}$ ). If the phase shift variation of a bunch is larger than $1^{\circ}$ to minimize the measurement error, the phase shift is extracted from a sinewave fit of the dipole synchrotron oscillations, as shown in Fig. 9.

Then, as the phase shift of each bunch is changing slowly during the time between bursts, the phase shift is smoothed by applying a local linear regression with a moving window of 10 bursts. As shown in Fig. 10 (red diamonds), after the post-processing the resulting phase shift is smoother.

In the new system, the turn-by-turn and bunch-by-bunch $(I, Q)$ data are transmitted by the phase module through a fiber optic link and stored in a circular buffer in a server known as ObsBox. This allows a larger number of turns to be acquired, increasing the precision and making the sine-wave fit optional.

\section{Experimental results}

The e-cloud buildup can be observed in Figs. 10 and 11 as a phase difference between the bunches at the beginning and the end of the bunch trains, shown for two fills during

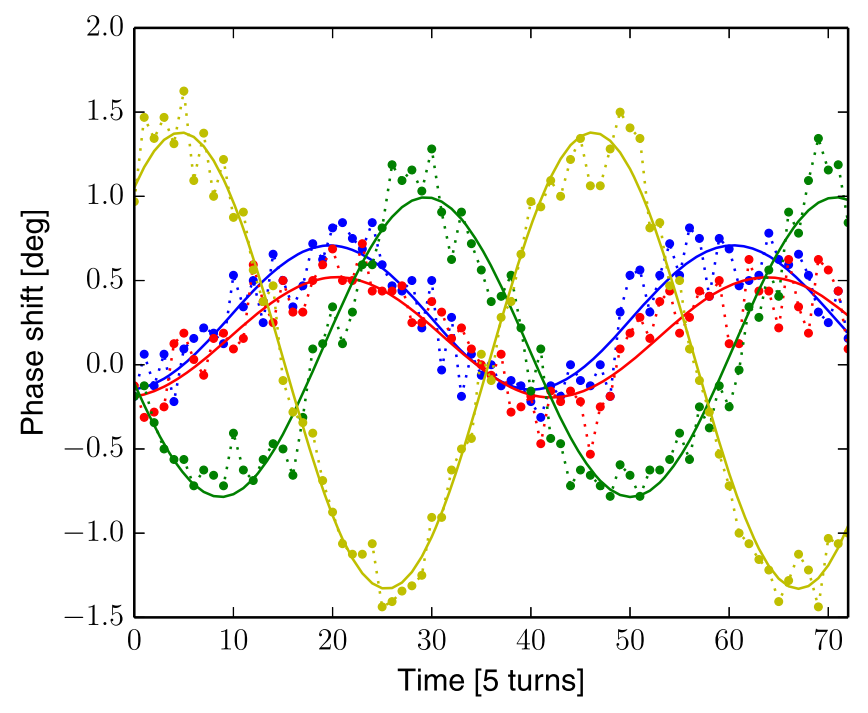

FIG. 9. Sine-wave fit (solid lines) of the synchrotron oscillations measured for four different bunches (dots) used in the data post-processing.

the 2012 scrubbing run with $25 \mathrm{~ns}$ beams. The reduction of the SEY due to scrubbing led to a decrease in the phase variation along the bunch trains from about $1.2^{\circ}$ at the beginning of the scrubbing run (Fig. 10) to $\sim 0.3^{\circ}$ at the end (Fig. 11). Nevertheless, the e-cloud effect was still significant for the $25 \mathrm{~ns}$ beams after the scrubbing run in 2012.

A similar effect could be seen before the 2011 scrubbing run for $50 \mathrm{~ns}$ beams, but no e-cloud was observed for these beams after reducing the SEY below the build-up threshold by scrubbing with beam. In 2012 the phase variation along the trains with $50 \mathrm{~ns}$ spacing was smaller than the measurement resolution (which is around $0.1^{\circ}$ ).

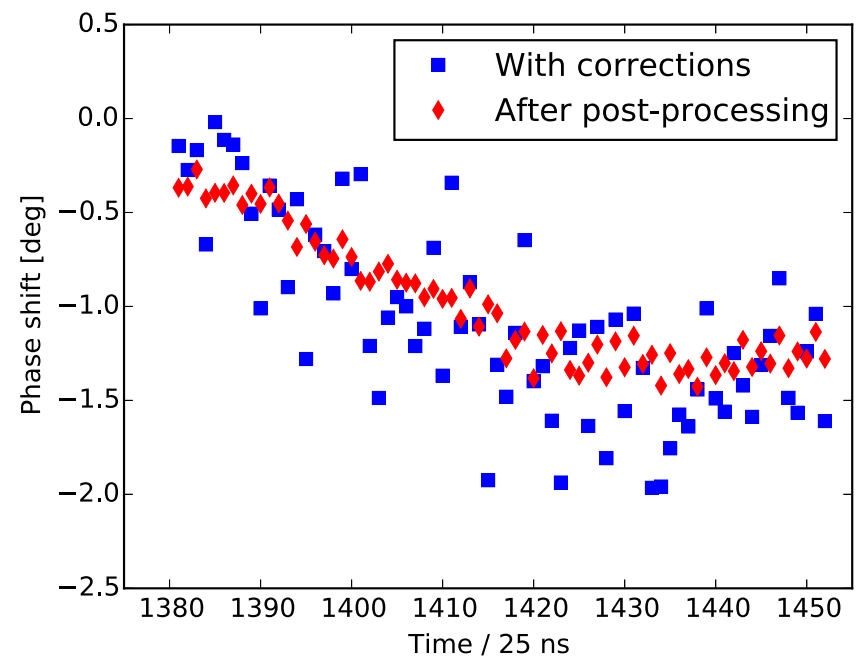

FIG. 10. Phase shift along the bunch train after corrections for systematic errors (blue squares) and after post-processing (red diamonds), applied to the same measurements shown in Fig. 3. 


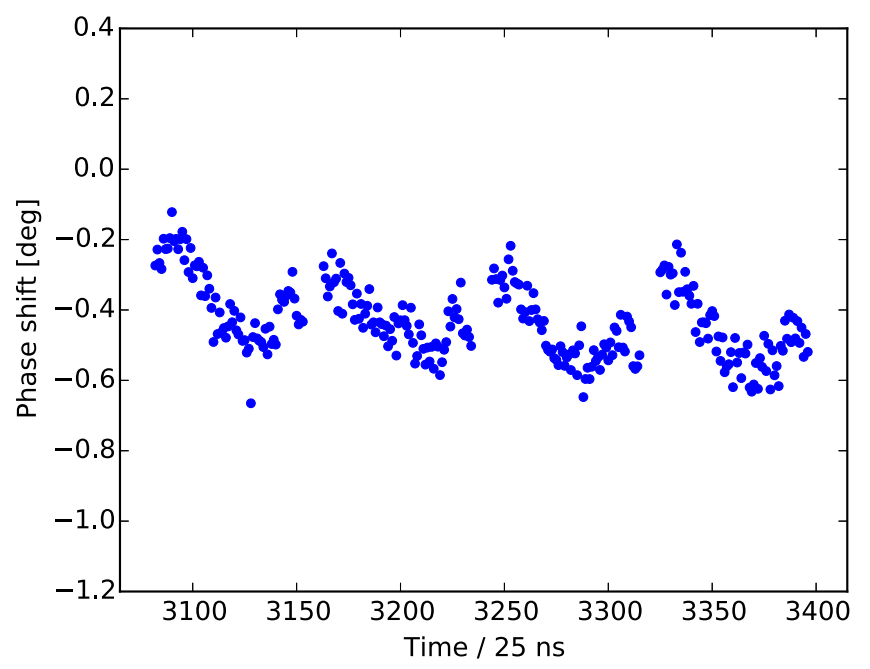

FIG. 11. Bunch-by-bunch phase shift (corrected and postprocessed) immediately after the injection of four trains of 72 bunches spaced by $250 \mathrm{~ns}$. Measurements on Beam 1 during a fill at the end of the 2012 scrubbing run with 25 ns beams (Fill 3405, 9-12-2012). $\bar{N} \sim 1.2 \times 10^{11}$.

Another observation of the e-cloud buildup is shown in Fig. 12 as an increasing bunch-by-bunch power loss along the bunch trains, calculated using Eq. (4) from the measured phase shift (corrected and post-processed). This example was measured before the 2012 LHC scrubbing run.

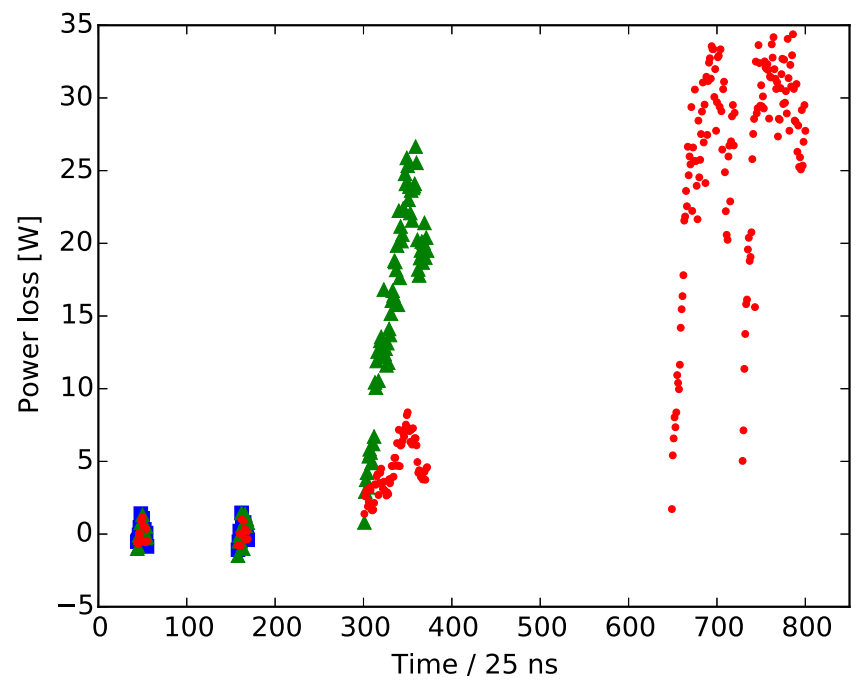

FIG. 12. Bunch-by-bunch power loss on Beam 1 for a fill with 25 ns beams before the 2012 scrubbing run (Fill 2826, 10-72012) at three different moments: $15 \mathrm{~min}$ after the injection of the first trains of 12 bunches (blue squares), a few seconds after the injection of a train of 72 bunches (green triangles), and a few seconds after the injection of two trains of 72 bunches spaced by $225 \mathrm{~ns}$ (red circles). The first short bunch train (12 bunches, blue) is used as a reference for the phase shift measurement. $\bar{N} \sim 1.1 \times 10^{11}$.
Using the bunch-by-bunch phase shift instead of the average phase shift, the total beam power loss $P_{T}$ can be calculated more accurately as the sum over all bunches. This parameter allows the time evolution of the e-cloud density in the ring during the cycle to be seen. An example of the variation of the total beam power loss during a fill with beams with $25 \mathrm{~ns}$ spaced bunches is shown in Fig. 13 (black line). Note that the power loss increases after each bunch train injection and then again during the acceleration.

Similarly to what is presented in Fig. 5, the maximum of the average power loss per particle $P_{p}$ gives an indication of the e-cloud activity during one fill and can be calculated as:

$$
P_{p}=\max \left(\frac{P_{T}}{\sum_{k=1}^{M} N_{k}}\right)
$$

A comparison of $P_{p}$ for different fills during the 2012 scrubbing run is shown in Fig. 14 and the decrease in power loss is a clear indicator of the SEY reduction (scrubbing). Note that the scrubbing is fast at the beginning and it is slower afterwards due to the reduction of the e-cloud density.

Figure 15 shows the evolution of the power loss $P_{p}$ during the first scrubbing run in 2015 with 50 and 25 ns beams. A fast conditioning is observed at the beginning of the scrubbing run with $50 \mathrm{~ns}$ beams. In the second period with 50 ns beams, the SEY was already below the e-cloud build-up threshold and no e-cloud is observed. For the $25 \mathrm{~ns}$ beams, there is no apparent reduction of the e-cloud activity because the beam parameters were continuously optimized to enhance the e-cloud buildup and therefore the scrubbing efficiency. In particular, the number of bunches

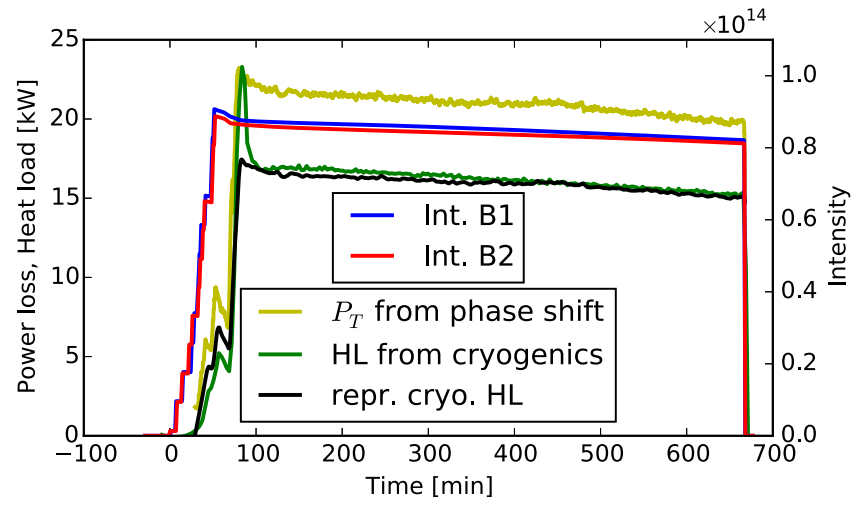

FIG. 13. Total beam power loss $\left(P_{T}\right)$ found from the phase shift (yellow line) and the heat load (HL) measured by the cryogenic system (green line) [13,14], for a fill with $25 \mathrm{~ns}$ beams accelerated to $4 \mathrm{TeV}$ (Fill 3429, 13-12-2012). An estimation of the cryogenic heat load (black line) was made from the phase shift measurements as described in the text (a scale factor of 0.76 was used to fit the part at $4 \mathrm{TeV}$ ). 


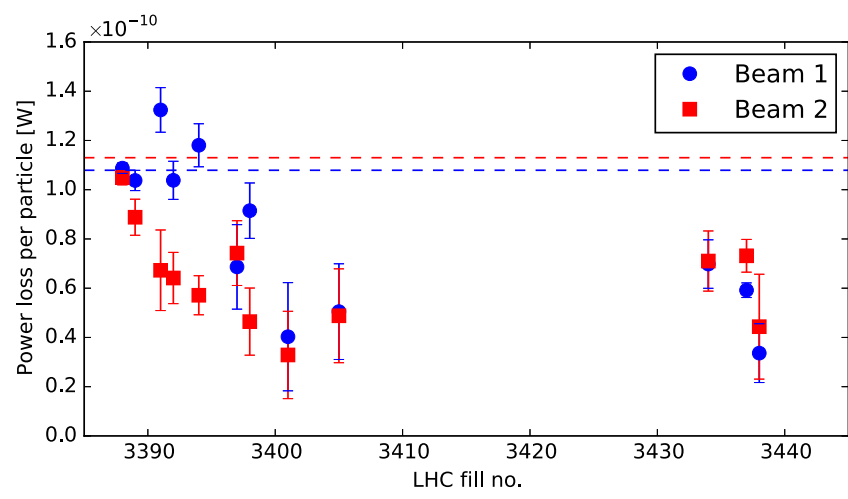

FIG. 14. Evolution of the maximum power loss per particle $P_{p}$ during the 2012 scrubbing run at $450 \mathrm{GeV}$ with $25 \mathrm{~ns}$ beams for Beam 1 (blue circles) and for Beam 2 (red squares). The dashed lines represent the level achieved after the scrubbing run in 2011 for both beams. The decrease in power loss is a clear indicator of the SEY reduction. Error bars are defined by the noise of the bunch-by-bunch phase measurements.

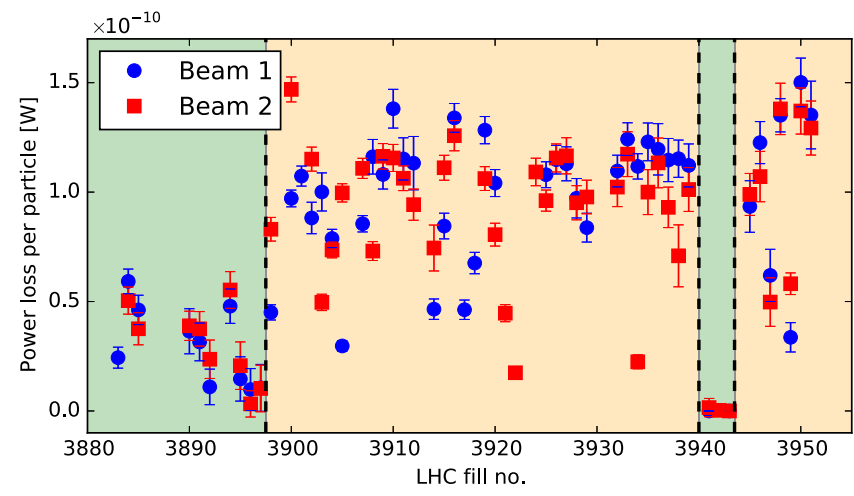

FIG. 15. Evolution of the maximum power loss per particle $P_{p}$ at $450 \mathrm{GeV}$ during the first scrubbing run in 2015 for Beam 1 (blue circles) and for Beam 2 (red squares). The areas shaded in green correspond to periods with $50 \mathrm{~ns}$ beams and those shaded in orange to $25 \mathrm{~ns}$ beams. The effect of scrubbing is only visible when the beam parameters are similar, as for first fills with $50 \mathrm{~ns}$ beams of for fills 3933-3939. Outliers are correlated with fills with lower intensity per bunch and/or small number of injections.

per injection was gradually increased (12-24-36-48$60-72$ bunches). For each case, the number of injections per fill was increased in steps and the spacing between bunch trains reduced. With these considerations, a reduction of the e-cloud activity can only be observed when conditions are similar, as for example for fills from 3933 to 3939 .

\section{COMPARISON WITH OTHER E-CLOUD OBSERVATIONS}

The e-cloud observation method described in this paper has been compared with an indirect measurement of the e-cloud density based on the heat load deposited in the cryogenic system [13,14], as well as with macroparticle simulations using the code PyECLOUD $[5,6]$.

\section{A. Cryogenic heat load measurements}

The energy lost by the beam due to the presence of e-cloud is transferred to the electrons and is finally deposited in the beam screens, where it has to be absorbed by the cryogenic system. Therefore, the beam power loss calculated from the phase shift can be compared with the heat load measured by the cryogenic system [13,14]. However, it is necessary to take into account that the cryogenic system sees the heat load only in the superconducting magnets in the arcs and, as the cryostat is shared by the two beam chambers, the contributions of both beams are added. Additionally, the cryogenic system has a slow time response $(\sim 5 \mathrm{~min})$ due to its large thermal inertia. Note also that the measured heat load includes image current and synchrotron radiation contributions, which should be calculated and subtracted to obtain an estimate of the e-cloud induced heating.

The heat load measurement by the cryogenic system can be reproduced by the beam power loss obtained from the measured phase shift by applying a moving average filter with a window of $5 \mathrm{~min}$, similar to the cryogenic system, and then defining a proper scale factor. An example of the estimation of the heat load in the cryogenic system from the phase shift for a fill with $25 \mathrm{~ns}$ beams accelerated to $4 \mathrm{TeV}$ is shown in Fig. 13. The scale factor giving the best agreement with the cryogenic heat load measurements (found for several fills in 2012) is $\sim 0.79$ at $4 \mathrm{TeV}$ and $\sim 0.7$ at $450 \mathrm{GeV}$. This means that the increase in the heat load with beam energy is larger in the arcs than in the straight sections, since the heat load in the cryogenic system due to e-cloud is determined by the arcs.

\section{B. Simulations}

The bunch-by-bunch power loss due to e-cloud has been calculated [6] from simulations performed with the code PYECLOUD (see [5] for details). In simulations, an energy balance is applied to a slice of the beam chamber to calculate the energy loss per turn of each bunch, defined as the difference between the total energy of the electrons before and after the bunch passage plus the energy lost in electron-wall collisions. This calculation is done for each different element of the accelerator and extrapolated to its full length.

Measurements taken in 2011 were selected for a comparison with simulations and they both are shown in Fig. 16. At that time, the power loss was dominated by the e-cloud in the dipole magnets and the effect from all other elements was neglected in the simulations. The simulations were using the measured beam parameters (filling pattern, bunch lengths, and intensities). The SEY was estimated from the measured heat load in the cryogenic system and found to be around 1.5. With this SEY value, 


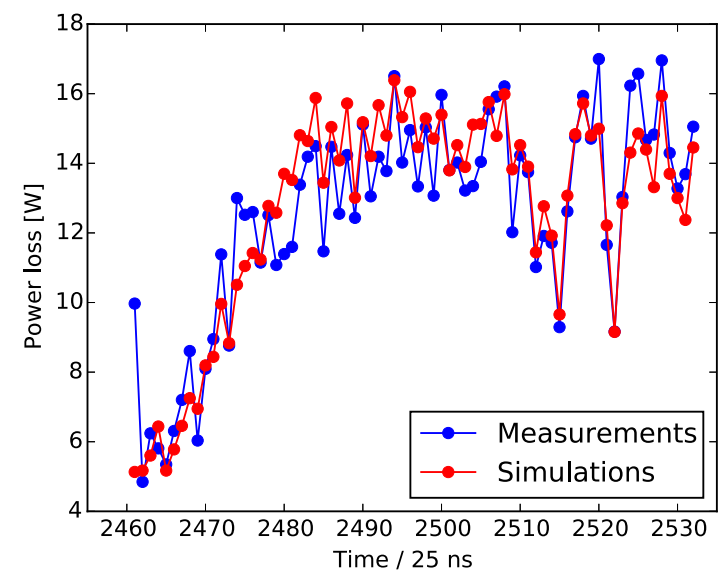

FIG. 16. Bunch-by-bunch energy loss per turn calculated from synchronous phase measurements for a bunch train of 72 bunches spaced by $25 \mathrm{~ns}$ (blue circles) and from simulations (red circles) based on the measured beam parameters [5,6]. $\bar{N} \sim 1.0 \times 10^{11}$. Fill 2251 (25-10-2011).

the agreement between measurements and simulations is good when the e-cloud is saturated (end of the bunch trains), but the buildup is faster in measurements. This discrepancy can be explained, for example, by the presence of uncaptured beam circulating in the machine. The simulation in Fig. 16 shows that by adding a $10 \%$ uncaptured beam the agreement is very good and the details of the e-cloud buildup seen in the phase shift measurements can be well reproduced. Nevertheless, this might be only a partial explanation, as the amount of uncaptured beam in the LHC is usually lower than $10 \%$ even during scrubbing runs. Another possible cause of the small discrepancy between the measurements and the simulated buildup could be due to the assumed SEY curve in the low energy region.

\section{LHC OPERATION}

During the LHC run 2, which started in April 2015, luminosity is produced using beams with 25 ns spaced bunches. A new diagnostic tool using the measured bunchby-bunch phase shift has been implemented in the LHC control room for operational purposes. It is being regularly used to monitor the e-cloud activity, first during the scrubbing runs (June and July, 2015) and then in operation during the intensity ramp-up with increasing number of bunches. The tool provides essential information for taking the decision on when to dump the beam and refill to achieve optimal scrubbing conditions and shorten the scrubbing run. The decision is based on the e-cloud activity seen in the measured bunch-by-bunch power loss.

A new system, described already in previous sections, is under development and will provide phase shift measurements with higher accuracy. A graphical user interface will be available at the CERN Control Centre (CCC) and the data will be stored in the LHC logging database.
Another possibility is to use the phase shift measurements as a feed-forward signal for the cryogenic system. As it was mentioned above, this tool provides instantaneous information on the heat load deposited by the e-cloud whereas the measured heat load in the cryogenic system has a time constant in the order of minutes. With the phase measurement information, necessary changes in the cryogenic system can be anticipated. To improve the accuracy of the predictions, a calibration of the scale factor for the heat load estimation can be done regularly using the measured heat load in the cryogenic system.

\section{CONCLUSIONS}

Bunch-by-bunch synchronous phase measurements have been suggested and proven to be a good diagnostic tool for the e-cloud effect. It is possible thanks to the high accuracy of the beam phase module and special corrections applied for signal treatment. This novel method can be used to observe the e-cloud build-up along the bunch trains and to calculate the total beam power loss. Measurements of the heat load in the cryogenic system are well reproduced, although a scale factor that depends on the beam energy needs to be applied. Phase shift measurements have a very good agreement with simulations of the e-cloud build-up. The use of this method in operation has been proven to ease the scrubbing run optimization and can be also used as an additional input for the cryogenic system.

\section{ACKNOWLEDGMENTS}

The authors would like to thank L. Tavian for providing measurements of the heat load in the cryogenic system. We are grateful to G. Rumolo and G. Iadarola for discussions and comparison of our measurements with simulations. Thanks to T. Bohl for his valuable advice. We would also like to thank the LHC OP team for their assistance during the measurements and G. Papotti for BQM tests.

\section{APPENDIX A: IN-PHASE AND QUADRATURE COMPONENTS}

A carrier of frequency $\omega$ modulated in amplitude and in phase by two narrow-band signals, respectively $A(t)$ and $\varphi(t)$, can be decomposed into two amplitude-modulated signals of the same frequency with a phase offset of $\pi / 2$ between them:

$$
\begin{aligned}
A(t) \cos [\omega t+\varphi(t)]= & A(t) \cos \varphi(t) \cos (\omega t) \\
& -A(t) \sin \varphi(t) \sin (\omega t) \\
= & I(t) \cos (\omega t)-Q(t) \sin (\omega t)
\end{aligned}
$$

where $I(t)$ and $Q(t)$ are called the in-phase and quadrature components. 


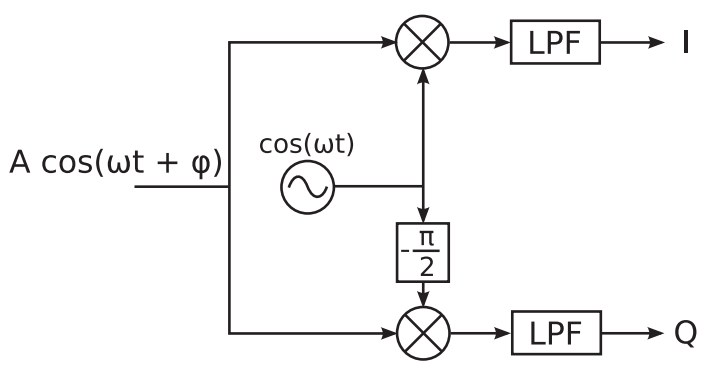

FIG. 17. Simplified scheme of an $I / Q$ demodulator. The input signal is split and mixed with two sine waves at the same frequency as the input signal and a phase offset of $\pi / 2$. These signals are low-pass filtered to remove the high-frequency mixing products.

A simplified scheme of an $I / Q$ demodulator is shown in Fig. 17. The input signal is split and mixed with two sine waves at the same frequency as the input signal and a phase offset of $\pi / 2$ between them. A low-pass filtering is required to remove the high-frequency mixing products at $2 \omega$. The $I(t)$ and $Q(t)$ components are, respectively, the result of the mixing with the sine wave at the same phase (in-phase) and with the sine wave shifted in phase by $-\pi / 2$ (quadrature). The $I(t)$ and $Q(t)$ signals are subsequently digitized and the amplitude $A(t)$ and the phase $\varphi(t)$ of the input signal can be easily calculated from the $I / Q$ components using the following relations: $A(t)=\sqrt{I(t)^{2}+Q(t)^{2}}$ and $\varphi(t)=\tan ^{-1}\left[\frac{Q(t)}{I(t)}\right]$.

\section{APPENDIX B: BEAM LOADING}

As mentioned in Sec. II, the synchronous phase measurements using the rf beam phase module should not be affected by beam loading. In order to confirm that statement, phase shift measurements were performed during a fill when the one-turn delay feedback of the low-level rf was switched on and off. The one-turn feedback is a system that reduces the transient beam loading and the effective impedance of the cavities by a factor $\sim 5$ [15]. Measurements were done during the commissioning of the one-turn feedback with 25 ns beams.

Measurements of the bunch-by-bunch phase shift and cavity phase are shown in Fig. 18. Although there is a clear reduction in the cavity phase modulation (due to beam loading) when the one-turn feedback was switched on, the variations observed in the phase shift are below the accuracy of the measurements $\left(\sim 0.1^{\circ}\right)$ and are most probably caused by changes in the beam parameters (bunch length and intensity) during the time between the measurements ( $\sim 30 \mathrm{~min})$. Therefore, the synchronous phase measurements are practically not affected by beam loading.

\section{APPENDIX C: BUNCH POSITIONS}

The beam quality monitor (BQM) is a system that uses longitudinal bunch profiles measured by a wall current monitor to determine longitudinal beam parameters during the LHC cycle [11]. One of them is the bunch center position, measured as the average of the two extreme values of the time at which the profile reaches half of its maximum value. The synchronous phase can be computed by applying a linear fit to the bunch positions, assuming that the distance between the buckets is constant ( $2.5 \mathrm{~ns})$.

Figure 19(a) shows an example of synchronous phase measurements using bunch positions. Although the sampling rate of the acquisition card used by the BQM is relatively low (8 GS/s), the precision of the measurements is improved by the interpolation which is applied in the algorithm that calculates the bunch position. Averaging over a few measurements also gives much cleaner results. However, the phase shift due to transient beam loading is included in these measurements and it is larger than the phase shift due to e-cloud. This can be seen when comparing the $\mathrm{BQM}$ measurements with measurements taken by the phase module at the same time and shown in Fig. 19(b). The estimation of the phase shift due to beam
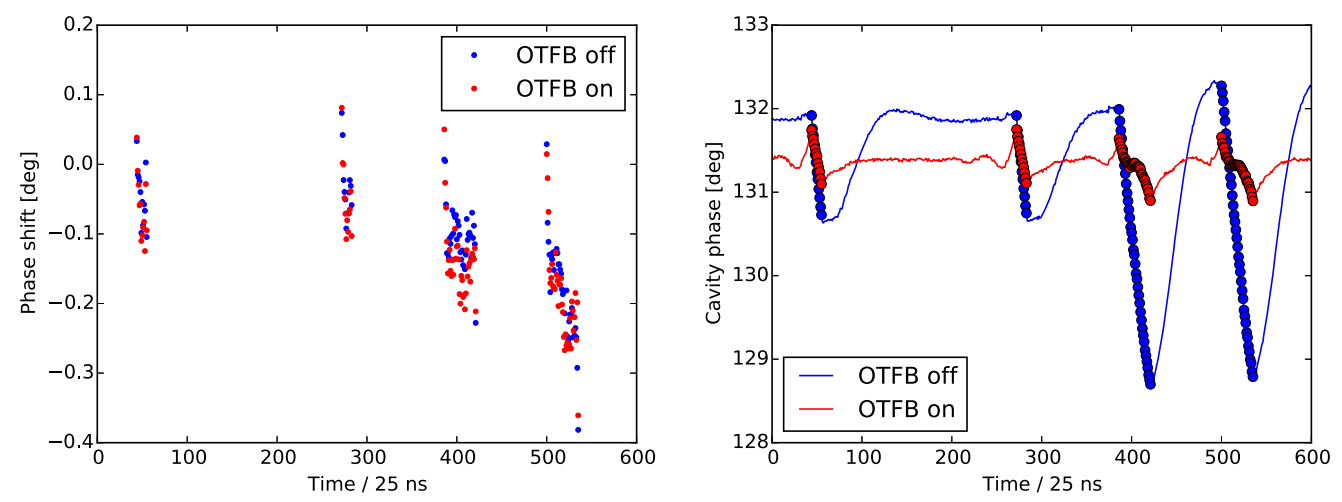

FIG. 18. Bunch-by-bunch phase shift (left) and cavity phase (right) with the one-turn feedback off (blue) and on (red). The circles on the right plot correspond to filled buckets. Beam 2. Fill 2248 (25 ns, 24-10-2011). 

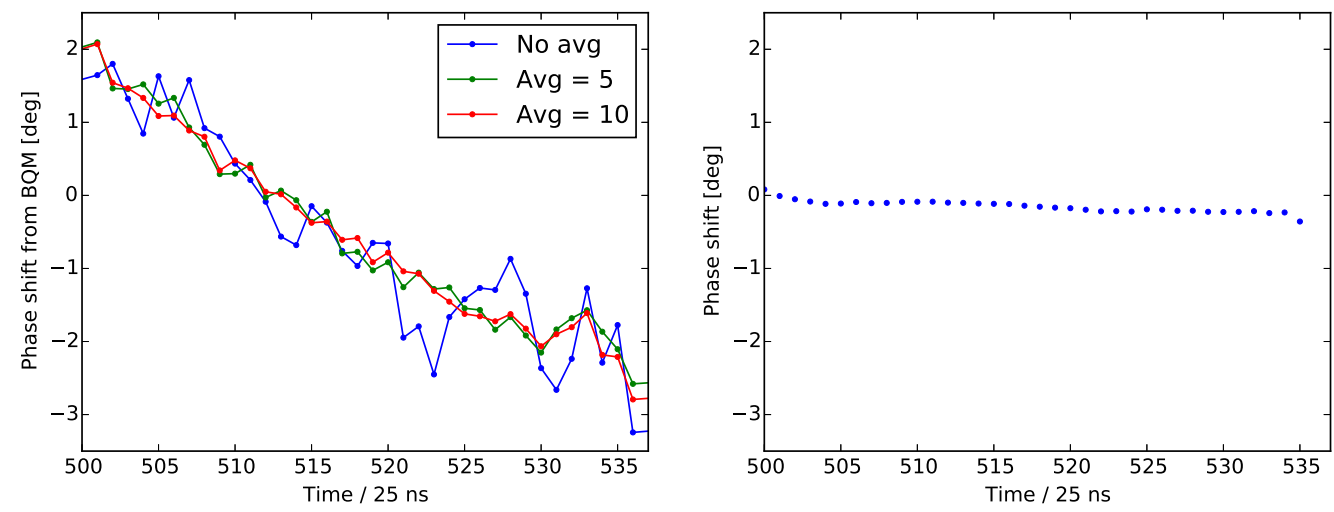

FIG. 19. Bunch-by-bunch phase shift computed from bunch positions measured by the BQM (left) and measured by the phase module (right). The larger phase shifts in panel (a) are due to beam loading with the one-turn feedback off. Beam 2 . Fill 2248 (25 ns, 24-10-2011).

loading effect with the required accuracy is very complicated and therefore it is difficult to extract the phase shift due to e-cloud from the measured bunch positions.

[1] F. Zimmermann, Review of single bunch instabilities driven by an electron cloud, Phys. Rev. ST Accel. Beams 7, 124801 (2004).

[2] G. Rumolo et al., Electron cloud observation in LHC, in Proceedings of the 2nd International Particle Accelerator Conference, San Sebastián, Spain (EPS-AG, Spain, 2011).

[3] R. Cimino, G. Rumolo, and F. Zimmermann, in Proceedings of Ecloud'12: Joint INFN-CERN-EuCARD-AccNet Workshop on Electron-Cloud Effects, ECLOUD'12, La Biodola, Italy, 2012 edited by R. Cimino, G. Rumolo, and F. Zimmermann (CERN, Geneva, 2013).

[4] G. Iadarola, G. Arduini, V. Baglin, H. Bartosik, O. Dominguez, J.F. Esteban Müller, G. Rumolo, E. Shaposhnikova, L. Tavian, and F. Zimmermann, Electron cloud and scrubbing studies for the LHC, in Proceedings of the 4th International Particle Accelerator Conference, IPAC-2013, Shanghai, China, 2013 (JACoW, Shanghai, China, 2013).

[5] G. Iadarola, Ph.D. thesis, Università degli Studi di Napoli Federico II, 2014.

[6] G. Iadarola and G. Rumolo, Electron cloud simulations with PyECLOUD, in Proceedings of the 11th International Computational Accelerator Physics Conference, ICAP2012, Rostock-Warnemnde, Germany, 2012 (JACoW, Germany, 2012).

[7] O. Boine-Frankenheim, E. Gjonaj, F. Petrov, F. Yaman, T. Weiland, and G. Rumolo, Energy loss and longitudinal wakefield of relativistic short proton bunches in electron clouds, Phys. Rev. ST Accel. Beams 15, 054402 (2012).

[8] J.F. Esteban Müller and E. Shaposhnikova, CERN Technical Report No. CERN-ACC-NOTE-2013-0007 PERF, 2012.
[9] D. Valuch and P. Baudrenghien, Beam phase measurement and transverse position measurement module for the LHC, in Low Level Radio Frequency Workshop (Knoxville, Tennessee, 2007).

[10] C. Roderick, L. Burdzanowski, and G. Kruk, The CERN accelerator logging service - 10 years in operation: a look at the past, present, and future, in Proceedings of the 14th International Conference on Accelerator \& Large Experimental Physics Control Systems, ICALEPS2013, 2013 (JACoW, San Francisco, 2013).

[11] G. Papotti, T. Bohl, F. Follin, and U. Wehrle, Longitudinal beam measurements at the LHC: The LHC beam quality monitor, in Proceedings of the 2nd International Particle Accelerator Conference, San Sebastián, Spain (EPS-AG, Spain, 2011).

[12] J. F. Esteban Müller, P. Baudrenghien, G. Iadarola, T. Mastoridis, G. Papotti, G. Rumolo, E. Shaposhnikova, and D. Valuch, Synchronous phase shift at LHC, in Proceedings of Ecloud'12: Joint INFN-CERN-EuCARDAccNet Workshop on Electron-Cloud Effects, ECLOUD'12, La Biodola, Italy, 2012 (CERN, Geneva, 2013).

[13] K. Brodzinski and L. Tavian, First measurements of beaminduced heating on the LHC cryogenic system, in Proceedings of the 24th International Cryogenic Engineering Conference and International Cryogenic Materials Conference, ICEC 24-ICMC 2012, Fukoaka, Japan, 2012 (Cryogenics and Superconductivity Society of Japan, Japan, 2013).

[14] L. Tavian, Performance limitations of the LHC cryogenics: 2012 review and 2015 outlook, in Proceedings of the 4th Evian Workshop on LHC beam operation, Evian-lesBains, France, 2012 (CERN, Geneva, 2013).

[15] T. Mastoridis, P. Baudrenghien, and J. Molendijk, LHC one-turn delay feedback commissioning, in Proceedings of the 3rd International Particle Accelerator Conference, New Orleans, LA, 2012 (IEEE, Piscataway, NJ, 2012). 